\title{
Identificación y Análisis de una Red de Ayuda Humanitaria. Un Caso de Estudio
}

\author{
Diana M. Gómez ${ }^{\star}$, William Sarache y Mariana Trujillo \\ Universidad Nacional de Colombia sede Manizales, Facultad de Ingeniería y Arquitectura, Departamento \\ de Ingeniería Industrial, Campus la Nubia, Km 4 Vía al Magdalena, Manizales, Caldas - Colombia. \\ (e-mail: dimgomezra@unal.edu.co,wasarachec@unal.edu.co, matrujilloga@unal.edu.co)
}

${ }^{*}$ Autor a quien debe ser dirigida la correspondencia.

Recibido Jul. 4, 2016; Aceptado Sep. 6, 2016; Versión final Nov. 4, 2016, Publicado Abr. 2017

\begin{abstract}
Resumen
Con el propósito de abordar diversos desafíos que enfrenta la logística humanitaria, se identificó la configuración de la red de ayuda humanitaria de la ciudad de Manizales, Colombia y luego se analizó utilizando la teoría de grafos como componente del análisis de redes sociales. La logística humanitaria enfrenta varios desafíos debido a las circunstancias adversas en las cuales se desarrolla. En particular, la coordinación entre actores ha sido identificada como uno de los mayores problemas cuando ocurre un desastre. La configuración de la red permitió determinar la estructura general y algunas características particulares de la red objeto estudio. Se identificaron tres actores de relevancia y la existencia de cuatro comunidades dentro de la estructura general de la red. Así mismo, se evidenció la baja densidad de la red, la cual constituye un aspecto importante a considerar en la formulación de políticas públicas en torno a las capacidades para la atención de desastres.
\end{abstract}

Palabras clave: logística humanitaria; ayuda humanitaria; gestión del riesgo de desastres; redes sociales; teoría de grafos

\section{Identification and Analysis of a Humanitarian Aid Network. A Case Study}

\begin{abstract}
With the purpose of addressing the several challenges that humanitarian logistics faces, the configuration of the humanitarian aid of Manizales city (Colombia) was identified and graph theory as a component of social network analysis was used. Humanitarian logistics faces these challenges due to adverse circumstances in which it takes place. In particular, coordination between actors has been identified as one of the biggest problems in this field of study. As a result, the overall structure and some particular network characteristics were determined. Also, the three most important actors and the existence of four communities were identified. The low network density was detected as one of the main problems, which constitutes an important aspect to consider in public policy formulation on disaster remediation.
\end{abstract}

Keywords: humanitarian logistics; humanitarian aid; disaster risk management; social networks; graph theory 


\section{INTRODUCCIÓN}

La logística humanitaria se define como "El proceso de planeación, implementación y control eficiente bajo un costo efectivo del flujo y almacenamiento de bienes y materiales, así como la información relativa, desde el punto de origen al punto de consumo con el fin de aliviar el sufrimiento de las personas vulnerables" (Thomas y Kopczak, 2005:2). Este proceso tiene lugar en las diferentes etapas que componen la gestión del riesgo de desastres, es decir, la mitigación, preparación, respuesta y recuperación. De otro lado, la logística humanitaria tiene como objetivo prevenir, atender y mitigar las consecuencias devastadoras ocasionadas por los desastres (Salazar et al., 2014; Leiras et al., 2014; Sheppard et al., 2013; Whiting y Ayala-Öström, 2009).

Dada la naturaleza de las actividades relacionadas con los desastres, la logística humanitaria se lleva a cabo en un ambiente complejo, caracterizado por la presión del tiempo y colmado de diversas fuentes de incertidumbre (Maghsoudi y Pazirandeh, 2016). A esta complejidad, se suma la presencia de múltiples actores ejecutando sus operaciones sobre el terreno, los cuales desarrollan diversas agendas que, muchas veces, afectan la coordinación necesaria para dar una respuesta eficiente (Chandes y Paché, 2009; Maghsoudi y Pazirandeh, 2016; Tatham y Spens, 2011).

Lograr una adecuada coordinación en la logística humanitaria, se ha convertido en un tópico de gran interés debido a su alto impacto en las operaciones humanitarias (Balcik et a., 2010), pues de esta depende el uso eficiente de recursos, los cuales, a su vez, definen el éxito o el fracaso de las cadenas de ayuda humanitaria (Balcik et al., 2010; Maghsoudi y Pazirandeh, 2016). Según Kaynak y Tuğer (2014), la coordinación entre actores se puede facilitar, empezando por el reconocimiento de todos aquellos involucrados en el proceso de logística humanitaria.

Por tal razón, el objetivo del presente trabajo fue identificar la configuración de la red de ayuda humanitaria, a partir de la determinación de los diferentes actores que la conforman. Para ello, se utilizó la teoría de grafos desde la perspectiva del análisis de redes sociales, pues ésta permite modelar las relaciones de un conjunto de actores para describir su estructura y funcionamiento. En atención a los aportes seminales de Wasserman y Faust (1994), la unidad de análisis consistió en una red conformada por un grupo de participantes (nodos) y los vínculos entre ellos (relaciones). La investigación se aplicó al caso de la ciudad de Manizales, la cual se encuentra ubicada en la región Centro-Occidental de Colombia, bajo una topografía abrupta y de alto riesgo (Younes et al., 2016). Dicha región, según el Programa de las Naciones Unidas para el Desarrollo (2005), está ubicada "en una de las áreas fisiográficas más interesantes del país, con mayor presencia de fenómenos naturales extremos y dinámicas sociales y ambientales propicias para la generación de riesgos" (p.12).

Para su presentación, el artículo ha sido estructurado de la siguiente manera: en la segunda sección se expone la metodología empleada para la identificación de la configuración de la red de ayuda humanitaria y el análisis de la misma; posteriormente, se presentan y discuten los resultados obtenidos en el caso de estudio $y$ finalmente, se plantea un conjunto de conclusiones

\section{METODOLOGÍA}

Con base en los aportes de Newman (2010), Scott (2000) y Wasserman y Faust (1994), los pasos ejecutados para identificar y analizar la configuración de la red de ayuda humanitaria, se resumen a continuación:

\section{Paso 1. Identificación de los actores de la red (nodos)}

Consiste en reconocer a cada uno de los participantes involucrados en las operaciones de ayuda humanitaria en determinado contexto; es decir, a nivel local, departamental o nacional. Este paso se desarrolló por medio de la consulta a diferentes fuentes de información, entre ellas, la normatividad nacional y local que regula la gestión del riesgo de desastres y la literatura especializada en el campo de estudio. Posteriormente, se sintetizaron los resultados encontrados y se derivó un listado de actores que permitió identificar la configuración de la red.

\section{Paso 2. Descripción de los vínculos entre actores (relaciones)}

Se debe determinar la existencia o ausencia de algún vínculo entre cada par de actores, a partir del estudio de las fuentes de información empleadas en la identificación de los mismos. Como resultado de lo anterior, se obtuvo un listado de las diferentes relaciones existentes, entre cada par de participantes de la red, por cada uno de los documentos consultados. 


\section{Paso 3. Valoración de las relaciones entre actores}

Implica asignar una magnitud a los diferentes vínculos reconocidos. Para ello, se otorga un valor numérico a cada una de las fuentes de información consultadas. La asignación de la valoración se puede realizar con base en la frecuencia de interacción entre un par de actores o a partir de la jerarquización de los documentos consultados, debido a la importancia que representa cada uno. En el primer caso, lo que se debe hacer es asignar pesos iguales a cada una de las fuentes de información y, en el segundo, se asignan pesos de acuerdo a la importancia de determinada fuente, frente a las demás. En cualquier caso, el peso de cada relación entre un par de participantes, estará dado por la sumatoria de los pesos de todos los documentos donde se identifique la misma.

\section{Paso 4. Representación gráfica de la red}

Por medio de un software visualizador de redes sociales y a partir de la información de relaciones entre nodos, se obtuvo la representación gráfica de la red bajo estudio.

\section{Paso 5. Análisis de la red}

Con base en los resultados gráficos obtenidos en el paso anterior, se realizaron tres tipos de análisis: (i) análisis estructural; (ii) análisis de centralidad del actor; y (iii) análisis por comunidades.

Análisis estructural: Para conocer la estructura general de la red, se evaluaron los indicadores de densidad y de diámetro, por medio de la ejecución de un software visualizador de redes sociales. La densidad indica la proporción de vínculos presentes entre los actores, frente al número total de conexiones posibles; tal medida toma valores entre cero y uno. Por su parte, el diámetro cuantifica la distancia más larga entre un par de actores en la red.

Análisis de centralidad del actor: Para determinar el actor más importante de la red, en el software visualizador de redes sociales, se obtuvieron las mediciones de los siguientes indicadores: 1) centralidad de grado: muestra el número de conexiones que tiene cada nodo en la red; 2) centralidad de vector propio: señala el prestigio de cada participante, a partir del cálculo de la importancia de los actores con los cual se relaciona; 3) centralidad de intermediación: indica la medida en que un actor se encuentra en el camino entre otros dos actores afectando la conexión de éstos últimos.

Análisis por comunidades: Permite reconocer patrones en las conexiones entre actores; dicho análisis se realizó identificando las diferentes sub-redes al interior de la red y evaluando, en cada una de ellas, los indicadores empleados en el análisis estructural y de centralidad del actor.

\section{RESULTADOS Y DISCUSIÓN}

Se presentan los resultados obtenidos en cada paso de la metodología propuesta, para el caso de estudio de la ciudad de Manizales.

\section{Paso 1. Identificación de los actores de la red (nodos)}

Los nodos corresponden a los diferentes actores encargados de la gestión del riesgo de desastres, definidos en el marco regulatorio vigente (Ley 1523 del 2012 del Congreso de la República, Decreto 200 del 2000 de la Alcaldía de Manizales y el Plan de Emergencias de Manizales 2003). También se incluyeron otros actores identificados en la literatura de logística humanitaria. En la Tabla 1 se presenta la lista completa de los 38 actores identificados y su correspondiente $l d$, el cual permite representar a cada actor en la gráfica de la red de ayuda humanitaria.

\section{Paso 2. Descripción de los vínculos entre actores}

En el caso de la red bajo estudio, los vínculos vienen dados por las siguientes consideraciones: 1) cada uno de los actores mencionados en el marco regulatorio sobre gestión del riesgo de desastres (Ley 1523 del 2012 y Decreto 200 del 2000), se relacionan con todos los demás actores identificados; 2) las relaciones de los actores identificados en el Plan de Emergencias de la ciudad bajo análisis, están dadas por su participación dentro de una misma actividad logística; y 3) los vínculos a incluir desde lo determinado en la literatura de logística humanitaria se exponen en la Tabla 2. 


\section{Paso 3. Valoración de las relaciones entre actores}

Está dada de acuerdo a la frecuencia de interacción entre un mismo par de actores. Para ello, se utilizó el método de ponderación de pesos iguales (Belhadj, 2012), donde a las relaciones identificadas en cada fuente de información se les asignó un mismo valor de 0.25 . De esta forma, el peso total de la relación entre un par de actores viene dada por la reiteración de la misma en las diferentes fuentes consultadas.

Tabla 1: Nodos que componen la red de ayuda humanitaria

\begin{tabular}{|c|c|c|c|}
\hline \multicolumn{4}{|c|}{ Actores en la red de ayuda humanitaria de Manizales } \\
\hline Id & Nombre del actor & Id & Nombre del actor \\
\hline 1 & Alcaldía & 20 & OMPAD \\
\hline 2 & COLPADE & 21 & INFIMANIZALES \\
\hline 3 & UNE & 22 & Hospitales \\
\hline 4 & CHEC & 23 & Secretaría de Desarrollo Comunitario \\
\hline 5 & Aguas de Manizales & 24 & Secretaría de Organización y Sistemas \\
\hline 6 & CORPOCALDAS & 25 & FRACOL \\
\hline 7 & Defensa Civil & 26 & Sociedad de Ingenieros y Arquitectos \\
\hline 8 & Cruz Roja & 27 & ONG's \\
\hline 9 & Bomberos & 28 & Otros actores interesados \\
\hline 10 & Secretario de Despacho Municipal & 29 & Secretaría de Hacienda \\
\hline 11 & Comandante de Policía & 30 & Control Interno \\
\hline 12 & Secretaría de Gobierno & 31 & Instituto de Cultura y Turismo \\
\hline 13 & Secretaría de Planeación & 32 & Voluntarios \\
\hline 14 & Secretaría de Obras Publicas & 33 & Aeronáutica Civil \\
\hline 15 & Secretaría de Salud & 34 & Proveedores de Logística \\
\hline 16 & Secretaría de Educación & 35 & Donantes \\
\hline 17 & Secretaría de Tránsito y Transporte & 36 & Iglesia \\
\hline 18 & Ejército Nacional & 37 & Medios de comunicación \\
\hline 19 & Terminal de transporte & 38 & Afectados \\
\hline
\end{tabular}

Tabla 2: Relaciones necesarias de acuerdo a la literatura de logística humanitaria

\begin{tabular}{|c|c|c|}
\hline Actor 1 & Actor 2 & Fuentes \\
\hline \multirow{7}{*}{ Iglesia } & Donantes & (Holguín-Veras et al., 2012) \\
\hline & Hospitales & (Holguín-Veras et al., 2012) \\
\hline & Cruz Roja & (Holguín-Veras et al., 2012) \\
\hline & Defensa Civil & (Holguín-Veras et al., 2012) \\
\hline & Voluntarios & (Holguín-Veras et al., 2012) \\
\hline & Afectados & $\begin{array}{l}\text { (Holguín-Veras et al., 2012; Van Wassenhove, } \\
\text { 2006) }\end{array}$ \\
\hline & ONG's & (Holguín-Veras et al., 2012) \\
\hline \multirow{7}{*}{ ONG's } & Proveedores de logística & $\begin{array}{c}\text { (Kovács y Spens, 2007; Pedraza et al., 2011; } \\
\text { Tomasini y Van Wassenhove, 2009; Vega y } \\
\text { Roussat, 2015) }\end{array}$ \\
\hline & Voluntarios & (Argollo et al., 2014) \\
\hline & Militares & (Argollo et al., 2014; Heaslip et al., 2012) \\
\hline & Gobierno & (Argollo et al., 2014) \\
\hline & Donantes & (Balcik et al., 2010) \\
\hline & Medios de comunicación & (Van Wassenhove, 2006) \\
\hline & Afectados & (Van Wassenhove, 2006) \\
\hline \multirow[t]{2}{*}{ Voluntarios } & $\begin{array}{l}\text { Organizaciones Humanitarias } \\
\text { (Cruz Roja y Defensa Civil) }\end{array}$ & (Falasca y Zobel, 2012) \\
\hline & Afectados & (Van Wassenhove, 2006) \\
\hline $\begin{array}{l}\text { Medios de } \\
\text { comunicación }\end{array}$ & Donantes & (Van Wassenhove, 2006) \\
\hline
\end{tabular}




\section{Paso 4. Representación gráfica de la red}

Por medio del software Ghepi (visualizador de redes sociales) y partir de la información sobre los actores y las relaciones establecidas entre ellos, se identificó el grafo de la red de ayuda humanitaria en Manizales (ver Figura 1). En esta, el área del círculo que representa a cada actor y la posición "central" del mismo, indica la importancia de cada actor dentro de la red en estudio. Así mismo, los colores identifican a la comunidad a la cual pertenece cada uno de ellos.

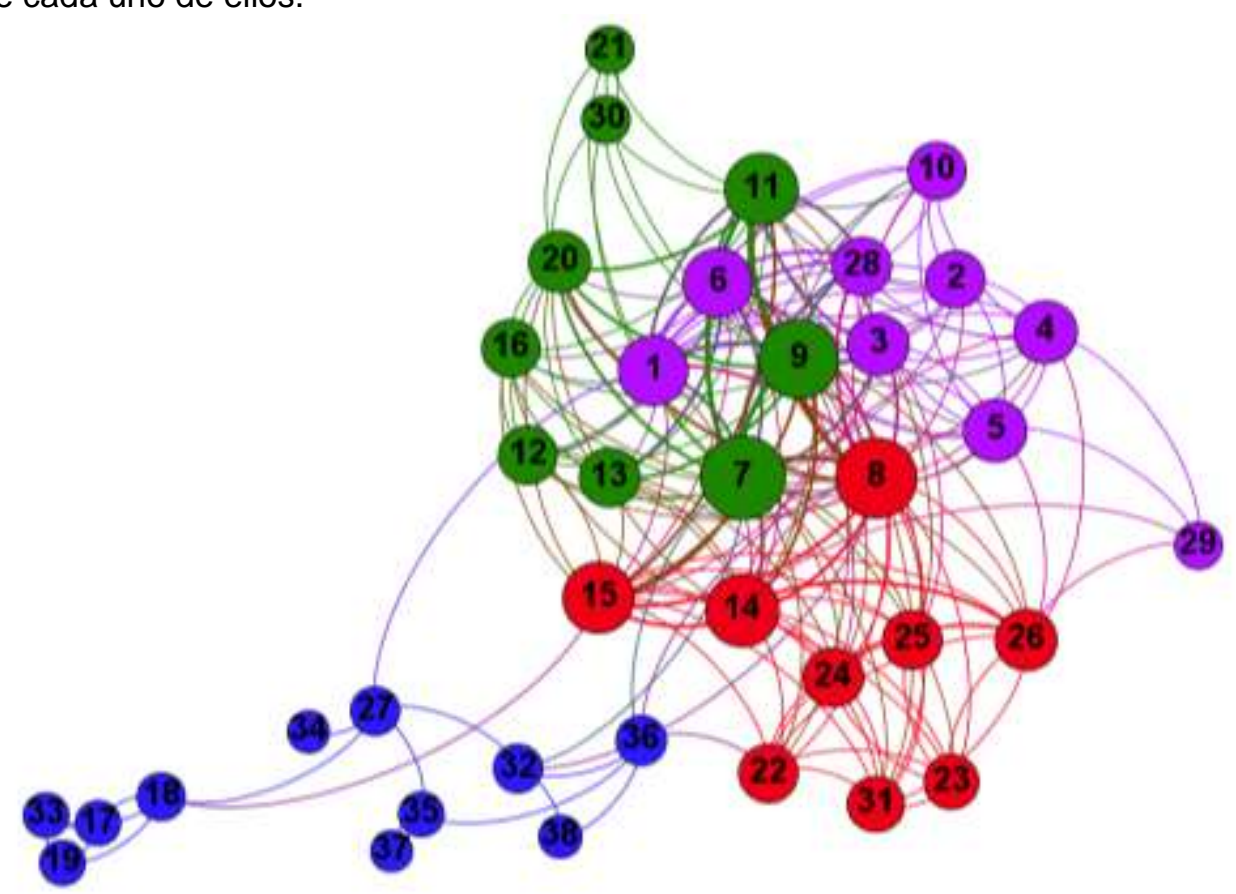

Fig. 1: Representación gráfica de la red de ayuda humanitaria en Manizales

Paso 5. Análisis de la red

Con la ayuda del software "Gephi", se obtuvo una serie de resultados que se presentan en diversas subsecciones.

\section{Análisis estructural}

La densidad de la red de ayuda humanitaria de Manizales, señaló que entre los 38 actores que componen la misma, se dan el $29 \%$ de los vínculos posibles. Para poder determinar si dicha densidad es alta, media o baja, se propone la escala de medición expuesta en la Tabla 3; la misma se basa en la proporción, según los planteamientos de Coronado Padilla (2007).

Tabla 3: Escala para la medición de la densidad

\begin{tabular}{|c|c|}
\hline Intervalos de valor & Categorías de la Densidad \\
\hline $0.80-1$ & Muy Alta \\
\hline $0.60-0.79$ & Alta \\
\hline $0.40-0.59$ & Media \\
\hline $0.20-0.39$ & Baja \\
\hline $0-0.19$ & Muy Baja \\
\hline
\end{tabular}

A partir de la escala propuesta, se puede determinar que la red tiene una densidad baja, lo cual indica que se trata de una comunidad poco cohesionada, donde el apoyo social es débil y es difícil la transmisión de información (Kadushin, 2012). Tal escenario ratifica un problema típico detectado en la literatura en relación a la dificultad en la coordinación entre actores (Chandes y Paché, 2009; Maghsoudi y Pazirandeh, 2016; Tatham y Spens, 2011).

Por su parte, el diámetro manifestó que los actores más distantes están separados por cinco conexiones; es decir, que entre ellos están presentes otros cuatro "actores intermediarios". La presencia de varios intermediarios interfiere en la comunicación (Wasserman y Faust, 1994), lo cual, a su vez, dificulta la coordinación (Balcik et al., 2010; Perry, 2007). 
Los indicadores de densidad y de diámetro de la red bajo estudio señalan la necesidad de proponer estrategias que busquen aumentar las relaciones entre los actores, para acotar la distancia en los caminos que separan a los participantes más distantes; esto, además de mejorar los indicadores estructurales de la red, permitirían aumentar la eficiencia de los esfuerzos en ayuda humanitaria adelantados en la ciudad objeto de estudio.

\section{Análisis de centralidad del actor}

Centralidad de grado: En Manizales, cada uno de los 38 actores que componen la red, están relacionados en promedio con otros 10; es decir, cada participante está vinculado con menos de la tercera parte de los actores, lo que corrobora la baja densidad de la red. En la tabla 4 se presentan los actores con más conexiones.

Tabla 4: Actores altamente conectados

\begin{tabular}{|l|c|}
\hline \multicolumn{1}{|c|}{ Actores } & $\begin{array}{c}\text { Grado de } \\
\text { conexiones }\end{array}$ \\
\hline Defensa Civil & 27 \\
\hline Cruz Roja & 25 \\
\hline Bomberos & 24 \\
\hline Comandante de policía & 20 \\
\hline Secretaría de Obras Públicas & 20 \\
\hline Secretaría de Salud & 19 \\
\hline
\end{tabular}

Centralidad de vector propio: Los actores con mayor valor de centralidad de vector propio se listan en la Tabla 5.

Tabla 5: Actores con mayor valor de centralidad de vector propio

\begin{tabular}{|l|c|}
\hline \multicolumn{1}{|c|}{ Actores } & $\begin{array}{c}\text { Valor de la centralidad } \\
\text { de vector propio }\end{array}$ \\
\hline Defensa Civil & 1 \\
\hline Cruz Roja & 0.971 \\
\hline Bomberos & 0.955 \\
\hline Comandante de Policía & 0.832 \\
\hline Secretaría de Obras Públicas & 0.827 \\
\hline CORPOCALDAS & 0.803 \\
\hline
\end{tabular}

Centralidad de intermediación: En la Tabla 6 se exponen los actores con mayor valor de intermediación, para el caso de Manizales.

Tabla 6: Actores con mayor valor de intermediación

\begin{tabular}{|l|c|}
\hline \multicolumn{1}{|c|}{ Actores } & $\begin{array}{c}\text { Valor de } \\
\text { intermediación }\end{array}$ \\
\hline Secretaría de Salud & 0.17 \\
\hline Ejército Nacional & 0.157 \\
\hline Defensa Civil & 0.149 \\
\hline ONG's & 0.108 \\
\hline Cruz Roja & 0.105 \\
\hline Alcalde & 0.095 \\
\hline Iglesia & 0.09 \\
\hline
\end{tabular}

A partir del análisis de centralidad, se pudo determinar que la Defensa Civil, la Cruz Roja y los Bomberos son los actores más importantes en la red de ayuda humanitaria en Manizales, puesto que reportan los valores más altos en los diferentes indicadores. Cabe mencionar que los mismos, se pueden catalogar como actores clave en la fase de respuesta a las emergencias debido a la naturaleza de las operaciones que desarrollan (Argollo et al., 2014; Van Wassenhove, 2006). Tal situación, permite identificar una fortaleza en la ciudad analizada.

\section{Análisis por comunidades}

Se identificaron cuatro comunidades o sub-conjuntos de actores, a partir de la ejecución del algoritmo de "Louvain" (Lambiotte et al., 2009). Tales comunidades se exponen en la Figura 2. 


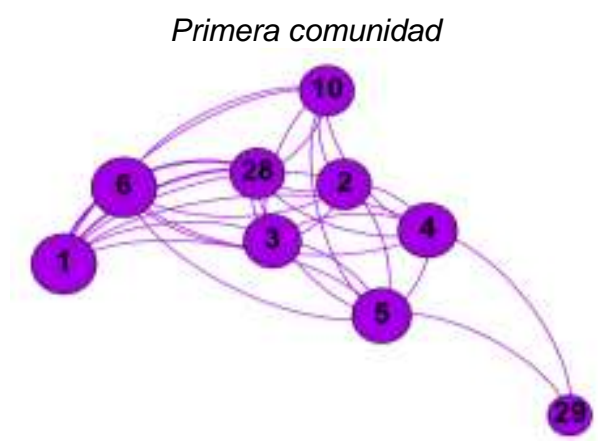

Tercera comunidad

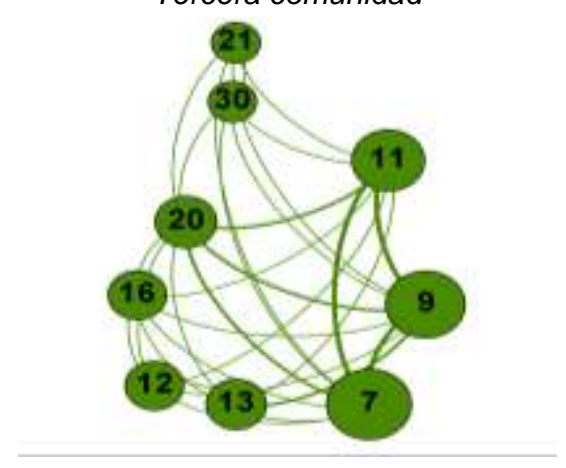

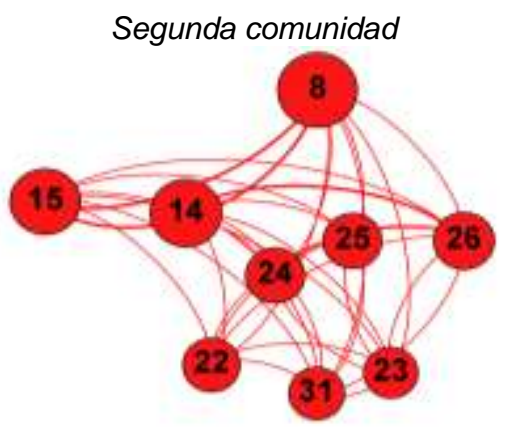

Cuarta comunidad

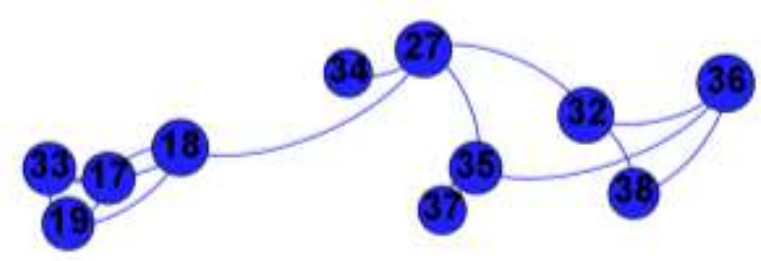

Fig. 2: Comunidades identificadas en la red de ayuda humanitaria de Manizales

Análisis de la primera comunidad: En esta comunidad se dan el $83 \%$ de las relaciones posibles, lo cual se considera como una densidad muy alta. Este resultado tiene su explicación en que 8 de los 9 actores que componen la sub-red, se encuentran definidos en la Ley 1523 del 2012 y, por tanto, en teoría todos se deben relacionar entre sí. Por otra parte, el grado medio indica que cada uno de los actores se relaciona en promedio con otros seis participantes de la red. Los indicadores asociados a la primera comunidad se exponen en la Tabla 7.

Tabla 7: Grado, intermediación y centralidad de vector propio de la primera comunidad

\begin{tabular}{|c|l|c|c|c|}
\hline Id & \multicolumn{1}{|c|}{ Actor } & Grado & Intermediación & $\begin{array}{c}\text { Centralidad } \\
\text { de vector propio }\end{array}$ \\
\hline 1 & Alcalde & 7 & 0.095 & 0.964 \\
\hline 2 & COLPADE & 7 & 0 & 0.964 \\
\hline 3 & UNE & 7 & 0.006 & 0.964 \\
\hline 4 & CHEC & 8 & 0.012 & 1 \\
\hline 5 & Aguas de Manizales & 8 & 0.012 & 1 \\
\hline 6 & CORPOCALDAS & 7 & 0.014 & 0.964 \\
\hline 10 & Secretaría de despacho municipal & 7 & 0 & 0.964 \\
\hline 28 & Otros actores interesados & 7 & 0 & 0.964 \\
\hline 29 & Secretaría de Hacienda & 2 & 0 & 0.283 \\
\hline
\end{tabular}

A pesar de que la mayoría de actores reportan indicadores similares, es importante destacar el papel de la empresa de energía de la ciudad (CHEC) y la empresa de acueducto (Aguas de Manizales), pues además de relacionarse con la totalidad de actores que conforman esta sub-red (valor del grado en la Tabla 7), son quienes administran los servicios básicos de la ciudad. Estos actores juegan un papel transcendente tanto en la respuesta a las situaciones de emergencia, como en la reconstrucción de una posible zona afectada.

Análisis de la segunda comunidad: Una densidad del 100\% con un valor de grado medio igual a 8, permiten determinar que en esta comunidad se dan la totalidad de relaciones posibles. Tal situación puede obedecer a que todos los actores que la componen están definidos en el plan de emergencias de la ciudad. Los indicadores relacionados con la tercera comunidad, se exponen en la Tabla 8. A partir de los resultados en los indicadores, se puede determinar que la Secretaria de Salud y la Cruz Roja, son los actores más importantes en la segunda comunidad debido a sus valores de intermediación. El hecho de que estos actores sean los más importantes, puede sugerir una fortaleza en el despliegue de la atención que requieran posibles víctimas, aspecto de gran relevancia en las situaciones de emergencia. 
Tabla 8: Grado, intermediación y centralidad de vector propio de la cuarta comunidad

\begin{tabular}{|l|l|c|c|c|}
\hline \multicolumn{1}{|c|}{ Id } & \multicolumn{1}{|c|}{ Actor } & Grado & Intermediación & $\begin{array}{c}\text { Centralidad de } \\
\text { vector propio }\end{array}$ \\
\hline 8 & Cruz Roja & 8 & 0.105 & 1 \\
\hline 14 & Secretaría de Obras Públicas & 8 & 0.039 & 1 \\
\hline 15 & Secretaría de Salud & 8 & 0.17 & 1 \\
\hline 22 & Hospitales & 8 & 0.016 & 1 \\
\hline 23 & Secretaría de Desarrollo Comunitario & 8 & 0 & 1 \\
\hline 24 & Secretaría de Organización y Sistemas & 8 & 0.003 & 1 \\
\hline 25 & FRACOL & 8 & 0.003 & 1 \\
\hline 26 & Sociedad de Ingenieros y Arquitectos & 8 & 0.016 & 1 \\
\hline 31 & Instituto de Cultura y Turismo & 8 & 0 & 1 \\
\hline
\end{tabular}

Análisis de la tercera comunidad: En esta, se dan el $83 \%$ de las relaciones posibles, lo cual es una densidad muy alta. Este valor obedece a que la totalidad de los actores están definidos en documentos locales que guían la atención de las emergencias humanitarias, como lo son el Decreto 200 de la Alcaldía de Manizales (2000) y el plan de emergencias de la ciudad. Por su parte, el valor de grado medio indica que cada uno de los actores se relaciona, en promedio, con otros seis participantes de la red (ver Tabla 9).

Tabla 9: Grado, intermediación y centralidad de vector propio de la tercera comunidad

\begin{tabular}{|l|l|c|c|c|}
\hline \multicolumn{1}{|c|}{ Id } & \multicolumn{1}{|c|}{ Actor } & Grado & Intermediación & $\begin{array}{c}\text { Centralidad de } \\
\text { vector propio }\end{array}$ \\
\hline 7 & Defensa Civil & 8 & 0.149 & 1 \\
\hline 9 & Bomberos & 8 & 0.054 & 1 \\
\hline 11 & Comandante de Policía & 8 & 0.032 & 1 \\
\hline 12 & Secretaría de Gobierno & 6 & 0 & 0.825 \\
\hline 13 & Secretaría de Planeación & 6 & 0.001 & 0.825 \\
\hline 16 & Secretaría de Educación & 6 & 0 & 0.825 \\
\hline 20 & OMPAD & 8 & 0.01 & 1 \\
\hline 21 & INFIMANIZALES & 5 & 0 & 0.685 \\
\hline 30 & Control Interno & 5 & 0 & 0.685 \\
\hline
\end{tabular}

De la tercera comunidad se pueden destacar dos aspectos: primero, se observaron valores bajos de intermediación, lo cual puede favorecer la comunicación y cooperación entre nodos; segundo, la presencia de dos de los tres actores más importantes de la red, es decir, la defensa civil y los bomberos. Estas propiedades permiten que se considere esta comunidad como la más relevante dentro de la red, lo cual, a su vez, la convierte en un objeto de estudio para indagar con más profundidad las dinámicas que allí se presentan para adoptar sus buenas prácticas en la totalidad de la red.

Análisis de la cuarta comunidad: Se observó un $27 \%$ de las relaciones posibles; es decir se presenta una densidad baja. Esto se debe a que la mayoría de sus actores se identificaron en la literatura de logística humanitaria, la cual no ha sido considerada en la formulación de política pública sobre la gestión del riesgo de desastres. Tal situación evidencia una brecha entre los avances propuestos desde la literatura de logística humanitaria y el estado de la práctica de la gestión del riesgo de desastres en Manizales. Por su parte, un grado medio de 3 confirma la baja densidad de esta comunidad. Los indicadores se exponen en la Tabla 10.

Tabla 10: Grado, intermediación y centralidad de vector propio de la cuarta comunidad

\begin{tabular}{|c|l|c|c|c|}
\hline \multicolumn{1}{|c|}{ Id } & \multicolumn{1}{|c|}{ Actor } & Grado & Intermediación & $\begin{array}{c}\text { Centralidad de } \\
\text { vector propio }\end{array}$ \\
\hline 17 & Secretaría de Tránsito y Transporte & 3 & 0 & 0.818 \\
\hline 18 & Ejército Nacional & 4 & 0.157 & 1 \\
\hline 19 & Terminal de transporte & 3 & 0 & 0.818 \\
\hline 27 & ONG's & 4 & 0.108 & 0.732 \\
\hline 32 & Voluntarios & 3 & 0.039 & 0.52 \\
\hline 33 & Aeronáutica Civil & 3 & 0 & 0.818 \\
\hline 34 & Proveedores de Logística & 1 & 0 & 0.242 \\
\hline 35 & Donantes & 3 & 0.057 & 0.46 \\
\hline 36 & Iglesia & 3 & 0.09 & 0.456 \\
\hline 37 & Medios de Comunicación & 1 & 0 & 0.159 \\
\hline 38 & Afectados & 2 & 0 & 0.338 \\
\hline
\end{tabular}


De la tabla 10 se puede destacar el papel del Ejercito Nacional, actor de gran importancia en la atención de emergencias (Balcik et al., 2010; Heaslip et al., 2012) y el rol desempeñado por la iglesia y las ONG's, quienes por su reputación e imagen de confianza ante la sociedad, tienen gran poder para convocar trabajadores voluntarios y recolectar donaciones (Argollo et al., 2014; Holguín-Veras et al., 2012).

\section{CONCLUSIONES}

El análisis estructural de la red de ayuda humanitaria de la cuidad de Manizales, permitió determinar que en la misma se presentan retos asociados a la coordinación entre actores, pues además de identificar menos del $30 \%$ de las conexiones posibles, los actores más distantes en la misma están conectados por un "camino largo" con cuatro actores intermediaros. Esta situación debe motivar la búsqueda de estrategias que incentiven la colaboración entre actores y, con ello, la mejora en la eficiencia de la ayuda humanitaria. Por su parte, dado a que la Defensa Civil, la Cruz Roja y los Bomberos son los actores más importantes en la red analizada, se reconoce una fortaleza en las operaciones de respuesta en la ciudad analizada.

Con respecto al análisis de comunidades, las tres primeras, conformadas en su mayoría por individuos identificados en los documentos y normativas locales y nacionales, son aquellas que presentan mayores valores en los indicadores de densidad y de grado medio. Tal situación puede suponer una adecuada articulación de esfuerzos, a partir de lo mandado en diferentes documentos normativos. En contraste, la comunidad cuatro, compuesta en su mayoría por actores identificados desde la literatura, es quien registra los valores más bajos en los indicadores de densidad y grado medio. Tal situación demuestra que, a pesar de que en la teoría sobre logística humanitaria se describen unas relaciones importantes entre los actores de esta comunidad, las mismas no están incluidas en lo que se ha formulado a nivel nacional y local para hacer frente al riesgo de desastres.

Los resultados obtenidos constituyen un primer acercamiento, tanto a la identificación de la configuración de la red de ayuda humanitaria de Manizales, como al conocimiento de su funcionamiento. Tales aspectos se pueden considerar de pertinencia en el campo de la logística humanitaria, pues, en la literatura consultada, no se encontraron antecedentes de estudios de este tipo. Por tal razón, resulta importante avanzar, en trabajos futuros orientados a refinar el análisis de la red, en torno a las interacciones e intensidades entre los actores que la conforman. Aun así, los resultados señalan una brecha importante entre los actores propuestos en la literatura especializada y los que han sido realmente involucrados en las normativas y la política pública que rigen la ciudad analizada.

\section{REFERENCIAS}

Argollo, S. R., Bandeira, B. A., Mello, L. C. y Campos, V., Humanitarian supply chain: an analysis of response operations to natural disasters, European Journal of Transport and Infrastructure Research, ISSN: 1567-7141, 14 (14), 290-310 (2014)

Balcik, B., Beamon, B. C., Krejci, C. C., Muramatsu, K. M. y Ramirez, M., Coordination in humanitarian relief chains: Practices, challenges and opportunities, International Journal of Production Economics, 126 (1), 22-34 (2010)

Belhadj, B., New weighting scheme for the dimensions in multidimensional poverty indices, Economics Letters, 116 (3), 304-307 (2012)

Cardona, O. D. y Consultores Gestión de Riesgos, Plan de emergencias de Manizales documento base (2003)

Chandes, J. y Paché, G., Pensar la acción colectiva en el contexto de la logística humanitaria: Las lecciones del sismo de Pisco, Journal of Economics, Finance and Administrative Science, 14 (27), 47-62 (2009)

Coronado-Padilla, J., Escalas o niveles de medición, Paradigmas, 2(2), 104-125 (2007)

Decreto N²00, 1-8, Alcaldía de Manizales, Colombia (2000)

Falasca, M. y Zobel, C., An optimization model for volunteer assignments in humanitarian organizations, doi:10.1016/j.seps.2012.07.003, Socio-Economic Planning Sciences, 46 (4), 250-260 (2012)

Heaslip, G., A. Sharif, M. y Althonayan, A., Employing a systems-based perspective to the identification of inter-relationships within humanitarian logistics, doi:10.1016/j.ijpe.2012.05.022, International Journal of Production Economics, 139 (2), 377-392 (2012)

Holguín-Veras, J., Jaller M. y Wachtendorf, T., Comparative performance of alternative humanitarian logistic structures after the Port-au-Prince earthquake: ACEs, PIEs, and CANs, doi:10.1016/j.tra.2012.08.002, Transportation Research Part A: Policy and Practice, 46 (10), 1623-1640 (2012) 
Kadushin, C., Understanding social networks. Theories, concepts and findings, $1^{\text {a }}$ edición, 1-240, Oxford University Press, New York, United States of America (2012)

Kaynak, R. y Tuğer, A.T., Coordination and collaboration functions of disaster coordination centers for humanitarian logistics, doi:10.1016/j.sbspro.2013.12.486, Procedia-Social and Behavioral Sciences, 109, 432-437 (2014)

Kovács, G. y Spens, K. M., Humanitarian logistics in disaster relief operations, doi: 10.1108/09600030710734820, International Journal of Physical Distribution \& Logistics Management, 37, 99-114 (2007)

Lambiotte, R., Delvenne, J. C. y Barahona, M., Laplacian dynamics and multiscale modular structure in networks (2009)

Leiras, A., de Brito, I., Queiroz Peres, E., Rejane Bertazzo, T. y Yoshida Yoshizaki, H. T., Literature review of humanitarian logistics research: trends and challenges, doi:10.1108/JHLSCM-04-2012-0008, Journal of Humanitarian Logistics and Supply Chain Management, 4 (1), 95-130 (2014)

Ley 1523, 1-80, Congreso de la República de Colombia, Colombia (2012)

Maghsoudi, A. y Pazirandeh, A., Visibility, resource sharing and performance in supply chain relationships: insights from humanitarian practitioners, doi: 10.1108/SCM-03-2015-0102, Supply Chain Management: An International Journal, 21 (1), 125-139 (2016)

Newman, M. E. J., Networks: An Introduction, $1^{\text {a }}$ edición, 1-740, Oxford University Press, New York, United States of America (2010)

Pedraza Martinez, A. J., Stapleton, O. y Van Wassenhove, L. N., Field vehicle fleet management in humanitarian operations: A case-based approach, doi:10.1016/j.jom.2010.11.013, Journal of Operations Management, 29 (5), 404-421 (2011)

Perry, M., Natural disaster management planning. A study of logistics managers responding to the tsunami, International Journal of Physical Distribution and Logistics Management, 37 (5), 409-433 (2007)

Programa de las Naciones Unidas para el Desarrollo. Guardianas de la Ladera. Una estrategia preventiva y de generación de ingresos a grupos vulnerables desde la alcaldía de Manizales (2005)

Salazar, F., Cavazos, J. y Vargas, G., Logística humanitaria: Un enfoque del suministro desde las cadenas agroalimentarias, doi: 10.4067/S0718-07642014000400007, Información Tecnológica, 25(4), 43-50 (2014)

Scott, J., Social network analysis. A handbook, $2^{a}$ edición, 1-205, SAGE Publications, London, Great Britain (2000)

Sheppard, A., Tatham, P., Fisher, R. y Gapp, R., Humanitarian Logistics : Enhancing the Engagement of Local Populations, Journal of Humanitarian Logistics and Supply Chain Management, 3 (1), 22-36 (2013)

Tatham, P. y Spens, K., Towards a humanitarian logistics knowledge management system, ISSN: 09653562, Disaster Prevention and Management, 20 (1), 6-26 (2011)

Thomas, A. S. y Kopczak, L. R., From logistics to supply chain management: the path forward in the humanitarian sector, Fritz Institute, 15, 1-15 (2005)

Tomasini, R. M. y Van Wassenhove, L. N., From preparedness to partnerships: case study research on humanitarian logistics, International Transactions in Operational Research, 16 (5), 549-559 (2009)

Van Wassenhove, L., Humanitarian aid logistics: supply chain management in high gear, The Journal of the Operational Research Society, 57 (5), 475-489 (2006)

Vega, D. y Roussat, C., Humanitarian logistics: the role of logistics service providers, doi: 10.1108/IJPDLM12-2014-0309, International Journal of Physical Distribution \& Logistic Management, 45 (4), 352-375 (2015)

Wasserman, S., Faust, K., Social network analysis: Methods and applications, $1^{\text {a }}$ edición, 1-825, Cambridge University Press, New York, United States of America (1994)

Whiting, M. C., Ayala-Öström, B. E., Advocacy to promote logistics in humanitarian aid, doi: 10.1108/01409170910998309, Management Research News, 32 (11), 1081-1089 (2009)

Younes, C., Escobar, D. A. y Holguín, J. M., Equidad, Accesibilidad y Transporte. Aplicación explicativa mediante un Análisis de Accesibilidad al Sector Universitario de Manizales (Colombia), doi: 10.4067/S071807642016000300010, Información Tecnológica, 27(3), 107-118 (2016) 